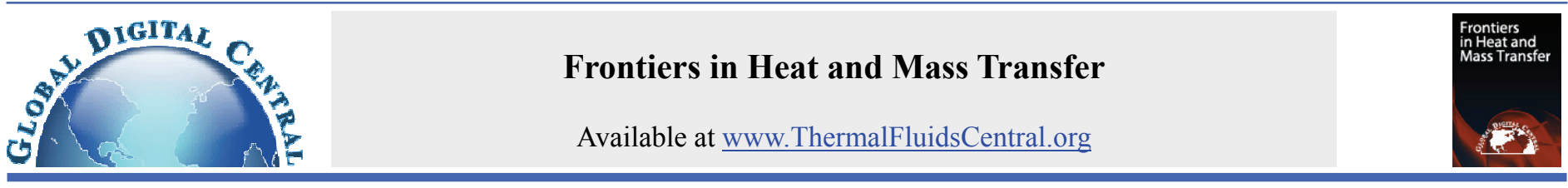

\title{
LIQUID WATER DYNAMIC BEHAVIORS IN THE GDL AND GC OF PEMFCS USING LATTICE BOLTZMANN METHOD
}

\author{
Li Chen, Hui-Bao Luan, Wen-Quan Tao* \\ School of Energy \& Power Engineering, Xi'an Jiaotong University, Xi'an, Shaanxi, 710049, China
}

\begin{abstract}
Multi-phase lattice Boltzmann method is applied to investigate liquid water transport in th GDL and GC. The liquid water transport processes in the GDL, near the GDL-GC interfaces and in the GC are discussed. The effects of channel land on liquid water dynamic behaviors and distribution in the GDL and GC are investigated. It is found that channel land covers the GDL-GC interface where liquid water reaches changes the water distribution near the GDL-GC interface and in the GC. While channel land is apart from the GDL-GC interface where liquid water reaches changes the effects of channel land is smaller.

Keywords: Liquid water; Channel land; GC; GDL; PEMFC
\end{abstract}

\section{INTRODUCTION}

Over the past few years, water management has stimulated an extensive study focus on liquid water transport behaviors and its effect on fuel performance, due to its significant importance to the successful operation and performance of PEMFC. Poor water management in a PEMFC results in either flooding phenomenon or membrane hydrations. When flooding phenomenon occurs, liquid water fills pores of cathode gas diffusion layer (GDL) and catalyst layer (CL) and hinders the effective transport of oxygen to the CL, leading to reactant starvation and performance loss ( $\mathrm{Li}$, Tang et al. 2008). Moreover, liquid water blocks the gas channel (GC), resulting in mal-distribution of oxygen and increase in parasitic pumping power to overcome the increased pressure drop. Therefore, efforts have been made to alleviate flooding phenomenon in PEMFC by devising better GC patterns such as serpentine and interdigitated GC patterns (Nguyen, Berning et al. 2004; Nguyen 1996). In addition, adding a micro porous layer (MPL) between CL and GDL also helps to reduce the negative effects of flooding (Wilson, Valerio et al. 1995).

An effective water management hinges on the fundamental and intensive understanding of liquid water behaviors in CL, GDL and GC. Currently, due to the development of more efficient PEMFC which can operate at higher power densities with higher water generation rates, understanding of liquid water transport phenomenon tends to become more important and urgent. Among water management issues, the liquid water transport behavior in the GDL is an important process as it influences liquid water amount in the CL as well as affects the transport of reactants from GC to CL. The GDL plays a critical role in the water management which maintains the delicate balance between water removal and membrane hydration (Li, Tang et al. 2008). Carbon-fiber based porous structure, such as carbon paper and carbon cloth are the most commonly used materials for the GDL. Fig. 1 shows the microstructure of the GDL. The GDL is naturally hydrophilic. In order to enhance water removal and thus to facilitate gas diffusion in the GDL, the GDL is usually treated with hydrophobic agent such as PTFE.

Some experimental technologies had been adopted to investigate liquid water in-plane and through-plane distribution, such as neutron image, NMR, X-ray techniques, optical technologies and so on (Bazylak 2009). However, due to the limits of spatial and temporal resolution, these technologies only can provide liquid water transport phenomenon at the upper layers of the GDL, leading to water dynamic microscale transport behaviors and steady and transient distribution inside the GDL still unavailable. Therefore, modeling and simulations efforts have been attempted to elucidate liquid water transport phenomenon inside the GDL. The water transport in GDL has been investigated using continuum two-phase models (Natarajan and Nguyen 2001; Siegel, Ellis et al. 2004; Wang, Wang et al. 2001; You and Liu 2002). Due to the lack of two-phase flow correlations required for closure of macroscopic continuum models, such as capillary pressure vs. saturation ( $P$ vs. $S$ ), permeability vs. saturation ( $K$ vs. $S$ ), simulations and modeling of water transport in PEMFC using continuum models usually adopts empirical corrections measuring in sand (Gostick, Fowler et al. 2006). The steady saturation distribution profiles along the GDL thickness direction predicted by continuum models generally present a convex shape which is doubtful. In addition, continuum models fail to take into account the influence of structural morphology because of the base on theory of volume averaging (Puneet K. Sinha, P.Mukherjee et al. 2007).

More fundamental models and simulations are required to fully understanding microscale liquid water transport behaviors in the GDL. Pore-network model and lattice Boltzmann method (LBM) are useful tools for this purpose. Several studied adopting pore-network model had been published to investigate the formation and distribution of liquid water in GDL (Nam and Kaviany 2003), to obtain the GDL

\footnotetext{
*Corresponding author. Email: wqtao@mail.xjtu.edu.cn
} 
specific $P$ vs. S data (Koido, Furusawa et al. 2008), to acquire liquid water transient distribution in GDL (Puneet K. Sinha, P.Mukherjee et al. 2007) and to study effects of GDL structures and characteristics on liquid water distribution (Lee, Nam et al. 2009). Compared to porenetwork model, LBM is also a useful numerical scheme to investigate water transport phenomenon in the GDL because of it's ability to simulate based on the real complex pore structure of GDL (Chen and Doolen 1998). Recently, several reported literatures employed LBM to simulate fluid flow and transport processes in PEMFCs. In the research of J. Park et al. (Park, Matsubara et al. 2007), LBM was applied to simulate single-phase flow in GDL made of carbon cloth. Effective permeabilities were calculated and they found that the fiber tow orientation strongly affected the permeabilities of the GDL, indicating the anisotropic permeabilities of GDL. In another literature of them (Park and Li 2008), multi-phase LBM was applied to simulate the unsteady behaviors of a liquid droplet passing through GDL made of carbon paper/carbon cloth. T. Koido et al. measured the relative permeability in water-saturated GDL by combining single-phase LBM and multiphase LBM (Koido, Furusawa et al. 2008). Using multiplerelaxation-time (MRT) LBM, H. Liang et al. studied the anisotropic permeabilities of a GDL made of carbon paper. Different in-plane and through-plane permeabilities were obtained (Hao and Cheng 2009). Using the simulated permeabilities, the relationship between porosity and permeabilities is determined in the form of empirical correction. Wang et al. (Mukherjee, Wang et al. 2009) adopted LBM to simulate drainage process in 3D hydrophobic and mixed-wet GDL.

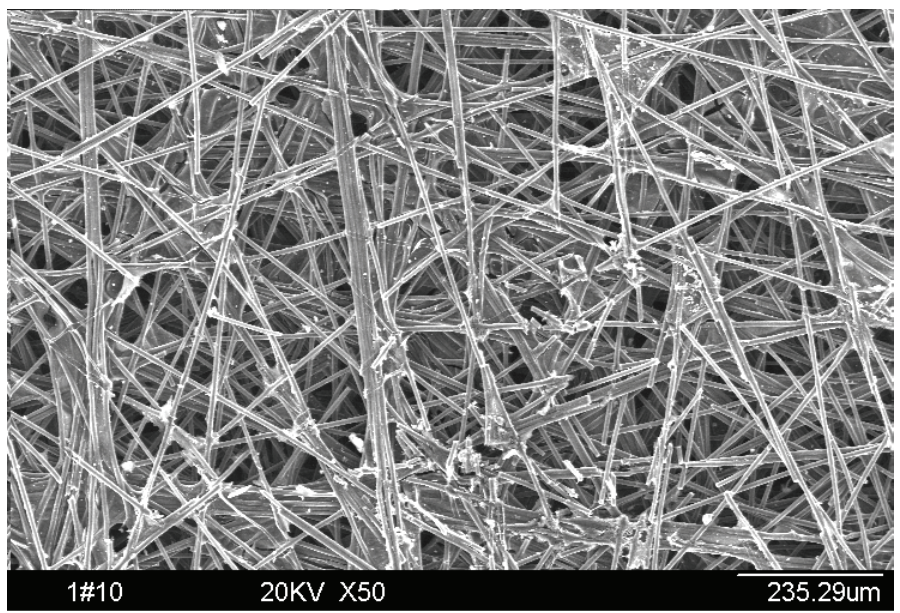

Fig. 1 Microstructure of carbon paper GDL

In the present paper, the Shan and Chen multi-phase lattice Boltzmann model (SC model) (Shan and Chen 1993; Shan and Chen 1996; Shan and Doolen 1995) is used to simulate liquid water microscale transport process in the GDL. The GC is also take into consideration and the effects of the channel land surface wettabilities and location on liquid water dynamic behaviors are investigated. Firstly, the SC model is described in Section 2. Then in section 3 the mathematical and physical model is introduced. In section 4 the results and discussion are presented. Finally, a conclusion is given in section 5 .

\section{MULTI-PHASE LB MODEL}

We first review SC model with inter-particle forces proposed by Shan and Chen (Shan and Chen 1993; Shan and Chen 1996; Shan and Doolen 1995). The interaction between particles is included in the kinetics through a set of potentials. The evolution LB equation for the $k$ th component is described by $f_{i}^{k}\left(x+\boldsymbol{e}_{i} \Delta t, t\right)-f_{i}^{k}(x, t)=-\frac{1}{\tau_{k}}\left(f_{i}^{k}(x, t)-f_{i}^{k, \mathrm{eq}}(x, t)\right)$

Where $f_{i}(x, t)$ is the number distribution function with velocity $\boldsymbol{e}_{i}$ at the lattice site $x$ and time $t$, and $\Delta t$ is the time increment. Superscript $k$ denotes the fluid component. $\tau$ is collision time which is related to the kinematical viscosity. $\boldsymbol{e}_{i}$ are the discrete velocities and for the D2Q9 model used in this paper, they are defined by

$$
\boldsymbol{e}_{i}= \begin{cases}0 & i=0 \\ \left(\cos \left[\frac{(i-1) \pi}{2}\right], \sin \left[\frac{(i-1) \pi}{2}\right]\right) & i=1,2,3,4 \\ \sqrt{2}\left(\cos \left[\frac{(i-5) \pi}{2}+\frac{\pi}{4}\right], \sin \left[\frac{(i-5) \pi}{2}+\frac{\pi}{4}\right]\right) & i=5,6,7,8\end{cases}
$$

For D2Q9 model, the equilibrium distribution function $f_{i}^{k, \mathrm{eq}}(x, t)$ is commonly chosen as

$$
f_{i}^{k, \mathrm{eq}}=w_{i} \rho_{k}\left[1+\frac{\boldsymbol{e}_{i} \cdot \boldsymbol{u}_{k}^{\mathrm{eq}}}{\left(c_{s}\right)^{2}}+\frac{\left(\boldsymbol{e}_{i} \cdot \boldsymbol{u}_{k}^{\mathrm{eq}}\right)^{2}}{2\left(c_{s}\right)^{4}}-\frac{\left(\boldsymbol{e}_{i} \cdot \boldsymbol{u}_{k}^{\mathrm{eq}}\right)^{2}}{2\left(c_{s}\right)^{2}}\right]
$$

For D2Q9 model, weight factor $w_{i}$ is $w_{i}=4 / 9, i=0 ; w_{i}=1 / 9, i=1,2,3,4$; $w_{i}=1 / 36, i=5,6,7,8 . c_{\mathrm{s}}$ is the speed of sound in the fluid and $c_{\mathrm{s}}=c / 3^{0.5}$ where $c$ equals $\Delta x / \Delta t$. Fluid density $\rho$ and fluid velocity $\boldsymbol{u}$ are obtained from the first and second moments of the distribution function

$$
\begin{aligned}
& \rho_{k}=\sum_{i} f_{i}^{k} \\
& \rho_{k} \boldsymbol{u}_{k}=\sum_{i} f_{i}^{k} \boldsymbol{e}_{i}^{k}
\end{aligned}
$$

In Eq. (3) $\boldsymbol{u}_{k}^{\text {eq }}$ is the macroscopic velocity and is given by the following relation

$$
\boldsymbol{u}_{k}^{\mathrm{eq}}=\boldsymbol{u}^{\prime}+\frac{\tau_{k} \boldsymbol{F}_{k}}{\rho_{k}}
$$

Where $\boldsymbol{u}^{\prime}$ is a common velocity for each component defined as

$$
\boldsymbol{u}^{\prime}=\frac{\sum_{k} \rho_{k} \boldsymbol{u}_{k} / \tau_{k}}{\sum_{k} \rho_{k} / \tau_{k}}
$$

$\boldsymbol{F}_{k}$ is the total force acting on the $k$ th component including fluid-fluid surface tension, fluid-solid adhesion and body forces. The total fluidfluid surface tension acting on the particles of the $k$ th component at lattice site $x$ is defined as (Shan and Chen 1993; Shan and Doolen 1995)

$$
\boldsymbol{F}_{c}^{k}=-\psi_{k}\left(\rho_{k}(x)\right) \sum_{x} \sum_{k}^{s} G_{k k}\left(x, x^{\prime}\right) \psi_{k}^{-}\left(\rho_{k}^{-}\left(x^{\prime}\right)\right)\left(x^{\prime}-x\right)
$$


The effective density $\psi_{k}\left(\rho_{k}\right)$ is defined as $\psi_{k}\left(\rho_{k}\right)=1-\exp \left(-\rho_{k}\right)$ in this paper. If only surface tension forces between the nearest and nextnearest neighbor points are considered, $g$, which controls the strength of the surface tension between different components, can be described as

$$
G_{k k}\left(x, x^{\prime}\right)= \begin{cases}4 g & \left|x-x^{\prime}\right|=1 \\ g & \left|x-x^{\prime}\right|=\sqrt{2} \\ 0 & \left|x-x^{\prime}\right|=0\end{cases}
$$

The fluid-solid interaction force $\boldsymbol{F}_{a}^{k}$ is introduced to describe the interaction between fluid of the kth component and wall (Martys and Chen 1996):

$$
\begin{aligned}
& \boldsymbol{F}_{a}^{k}=-\psi_{k}\left(\rho_{k}(x)\right) \sum_{x^{\prime}} W\left(x, x^{\prime}\right) s\left(x^{\prime}\right)\left(x^{\prime}-x\right) \\
& W\left(x, x^{\prime}\right)=\left\{\begin{array}{cc}
4 w & \left|x-x^{\prime}\right|=1 \\
w & \left|x-x^{\prime}\right|=\sqrt{2} \\
0 & \left|x-x^{\prime}\right|=0
\end{array}\right.
\end{aligned}
$$

$s$ is an indicator function and equal 0 or 1 for a pore or a solid, respectively. $w$, which controls the strength between fluid and wall, is positive for non-wetting fluid and negative for wetting fluid. By adjusting $w$, we can get different wetabilities.

\section{PHYSICAL MODEL AND LB SIMULATION}

\subsection{Computational domain}

3D reconstruction of microstructure of carbon paper GDL is performed by some previous work based on the statistical information from the realistic structure (Hao and Cheng 2009; Mukherjee, Wang et al. 2009). In the process of reconstruction, several assumptions are made for simplification, including straight fibers, fixed fibers diameters, negligible fibers oriented to the direction of the GDL thickness and randomly distributed intersecting carbon fibers in the in-plane direction. Then the reconstruction process can be implemented by giving the fiber diameter, porosity and GDL thickness. In this paper, the fibers diameter is set to be $7 \mu \mathrm{m}$, the porosity is equal to 0.8 and the thickness is set as $180 \mu \mathrm{m}$. Fig. 2(a) shows the reconstructed carbon paper GDL. Fig. 2(b) is a through-plane cross section of the 3D reconstructed GDL, in which a $500 \mu \mathrm{m}$ high GC is added at the top of the GDL. In order to alleviate effects of carbon fiber on the boundaries, five more lattices without carbon fibers are added to the left boundary and right boundary. Similarly, five more lattices are added to bottom boundaries. The final computational domain is $810 \mu \mathrm{m} \times 680 \mu \mathrm{m}$. In lattice units, the mesh is $810 \times 680$, meaning that the physical length of each lattice is $1 \mu \mathrm{m}$.

\subsection{Initial and boundary conditions}

At the bottom boundary, liquid water is injected into the GDL from $x=350 \mu \mathrm{m}$ to $\mathrm{x}=450 \mu \mathrm{m}$ with a given velocity. For the solid wall at the bottom boundary, no slip boundary condition is adopted. For the left and right boundary, periodic condition is applied. At the top boundary (the top wall of the GC) no slip boundary condition is adopted. In the GDL, because the liquid water transport mechanism is capillary fingering and capillary force is the dominant force, it is reasonable to set the density and viscosity of liquid water and air equal to each other when using SC model (Mukherjee, Wang et al. 2009; Park and Li 2008). Therefore, the densities and viscosities of liquid water and air in this paper are equal to each other. The relaxation time corresponding to viscosity is set as 1 for liquid water and air. In LBM, the velocity boundary condition is adopted to achieve the given velocity (Zou and He 1997). Bounce-back boundary condition is employed to achieve the non-slip boundary condition (Ziegler 1993).

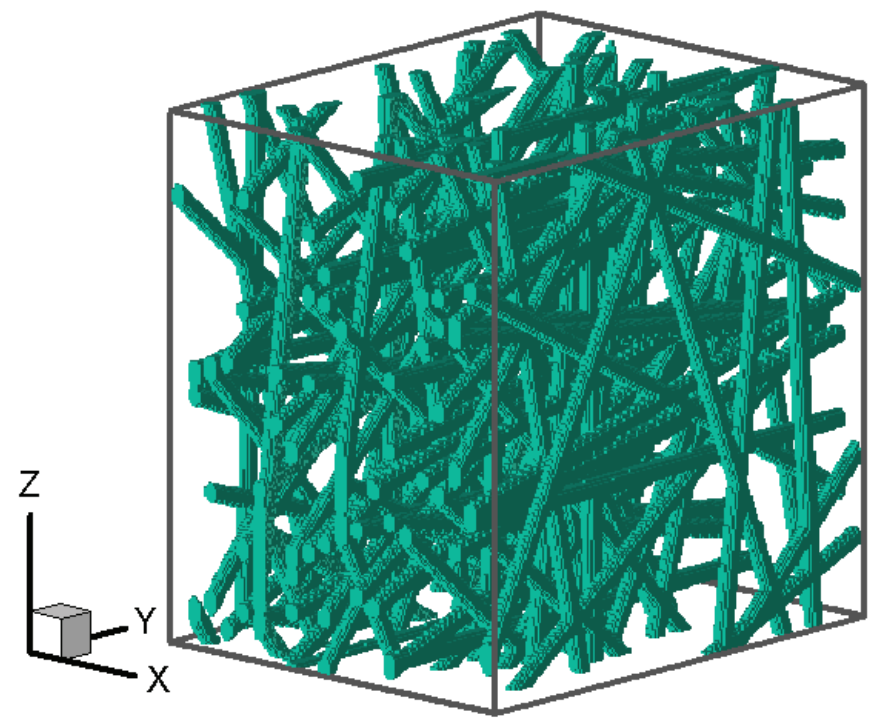

(a)

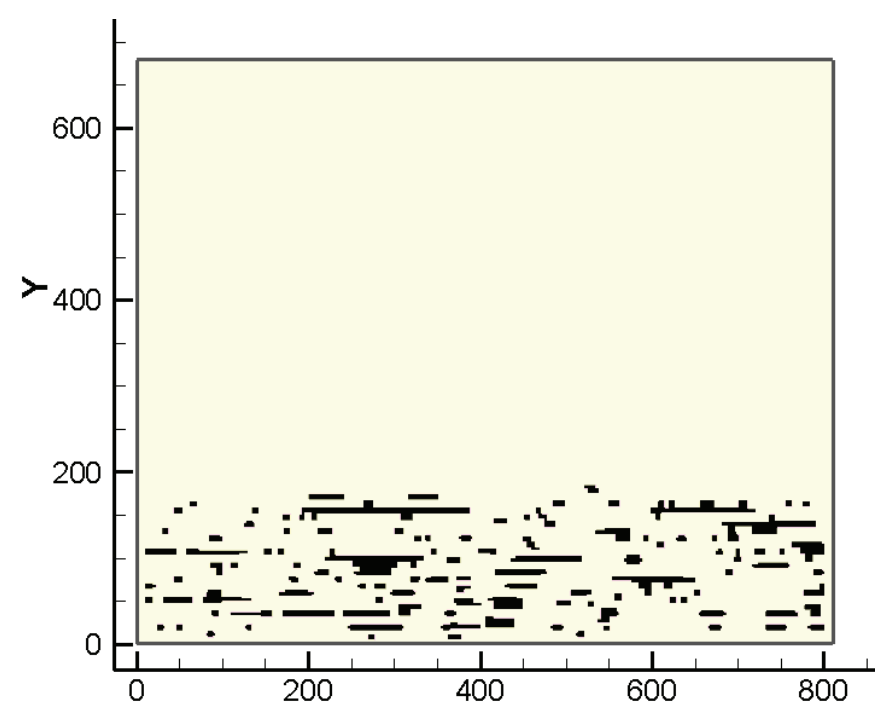

(b)

Fig. 2 (a) 3D reconstructed structure of GDL. (b) 2D computational domain contained GDL and GC

\subsection{Evaluation of surface tension}

Before investigating liquid water transport behaviors in GDL and GC, two validation cases are performed to verify that the codes developed in this paper can predict proper physical behaviors as well as determine some physical variables needed in the two-phase simulations, including $g$ that controls fluid-fluid interaction strength and $w$ that controls strength between fluid and wall.

In the SC model, different fluid-fluid interaction strength, namely the surface tension, can be obtained by adjusting $g$. If $g$ is small, components will not separate from each other and will mix with other. 
The mixed fluids separate from each other only if $g$ exceeds a critical value (Martys and Chen 1996). In order to validate this phenomenon a circular liquid droplet with radius of 20 lattices is put in the center of a $100 \times 100$ lattice system. Initial density of fluid 1(the liquid) is set as 2.0 inside the droplet and 1.0E-5 outside the droplet, respectively, and initial density of fluid 2 (gas) is set as $1.0 \mathrm{E}-5$ and 2.0 inside and outside the droplet, respectively. Periodic boundary conditions are applied in all the four directions. Physically the thickness of the interface between two fluids is on the order of angstroms. However, the thickness is not obtainable using LBM or other numerical methods. Usually the thickness of the interface between two fluids is several lattice units in LBM simulation (Martys and Chen 1996). Therefore a criterion is needed to distinguish the two fluids. In this paper we choose a simple criterion setting the cutoff density between the two fluids as 1.0.

Figure 3 shows the droplet inside the air. The difference between the maximum and the minimum density of fluid 1 related to $g$ is illustrated in Fig.4. It can be seen that the density difference is about zero, indicating that liquid and gas is miscible when $g$ is less than 0.25 . After this value as $g$ increases, the density difference becomes larger and larger implied the droplet is more pure. However, too large $g$ leads to a droplet density greater than 2.0 as shown in Fig.4. Therefore, in the following simulation $g$ is set as 0.5 .

Surface tension in lattice unit can be obtained from the Laplace's Law, which states that the pressure difference between the pressure inside the droplet $p_{\mathrm{i}}$ and the pressure outside the droplet $p_{\mathrm{o}}$ is related to surface tension $\sigma$ and droplet radius $\mathrm{R}$ by

$$
p_{i}-p_{o}=\sigma / R
$$

A series of numerical experiments are performed with different liquid water droplet radius. $p_{\mathrm{i}}$ and $p_{\mathrm{o}}$ are calculated 8 lattice away from the droplet surface as shown in the dashed red circles in Fig. 3. The pressure value is averaged around the circle. Fig. 5 shows the reciprocal of $R$ as a function of the pressure difference $\Delta p$. The pressure differences $\Delta p$ is indeed inversely proportional to the reciprocal of droplet radius. Least-square linear fit equation of the simulation results is $\Delta p=0.333 / R$, showing good agreement with Laplace's Law. The surface tension in lattice unit is 0.333 , which is the slope of the fitted line. The excellent agreement between the LBM simulation results and Laplace's Law proves that code developed can be used to conduct the following simulations.

\subsection{Evaluation of static contact angle}

Contact angle is considered as a measure of the solid surfaces wettability. The surface condition is wetting or hydrophilic if the contact angle is less than $90^{\circ}$, and the liquid has a tendency to spread on the solid surfaces. For hydrophobic surface or non-wetting surface, the contact angle is greater than $90^{\circ}$ and the liquid tends to form a droplet. We carry out simulations of a set of static semicircular droplets on a horizontal solid surface with $w$ changing from -0.2 to 0.2 to obtain different contact angle. In these simulations, the droplet initially is semicircular. Similar to simulation of evacuation of surface tension, initial density of fluid 1 is set as 2.0 inside the droplet and $1.0 \mathrm{E}-5$ outside the droplet, respectively. Initial density of fluid 2 is set as $1.0 \mathrm{E}-$ 5 and 2.0 inside and outside the droplet, respectively. Periodic boundary conditions are applied at the left and right boundary, at the bottom and top boundary non-slip boundary condition is adopted. No body force is applied. Once the steady state is reached, the radius of the droplet is calculated by the method proposed in (FAL. 1992).

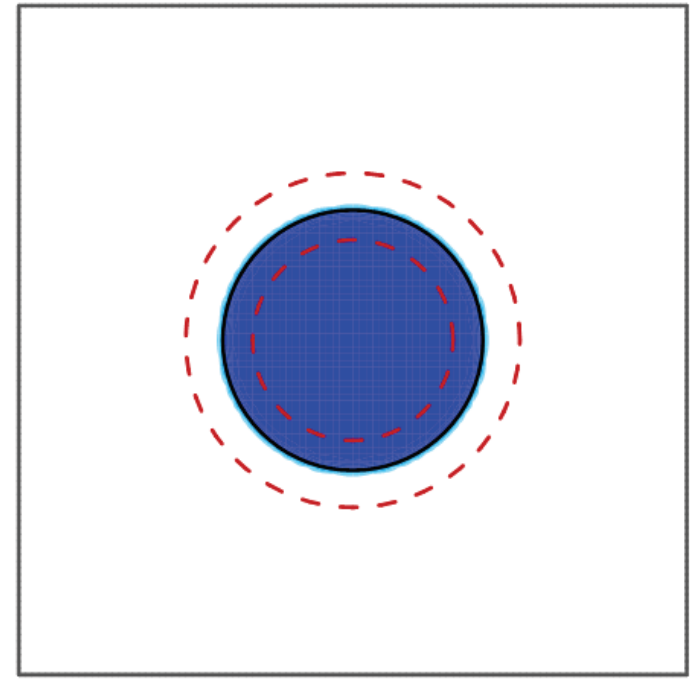

Fig. 3 A droplet in the air. Dash red circles are where the pressures inside and outside the droplet are calculated (8 lattices away from the droplet surface)

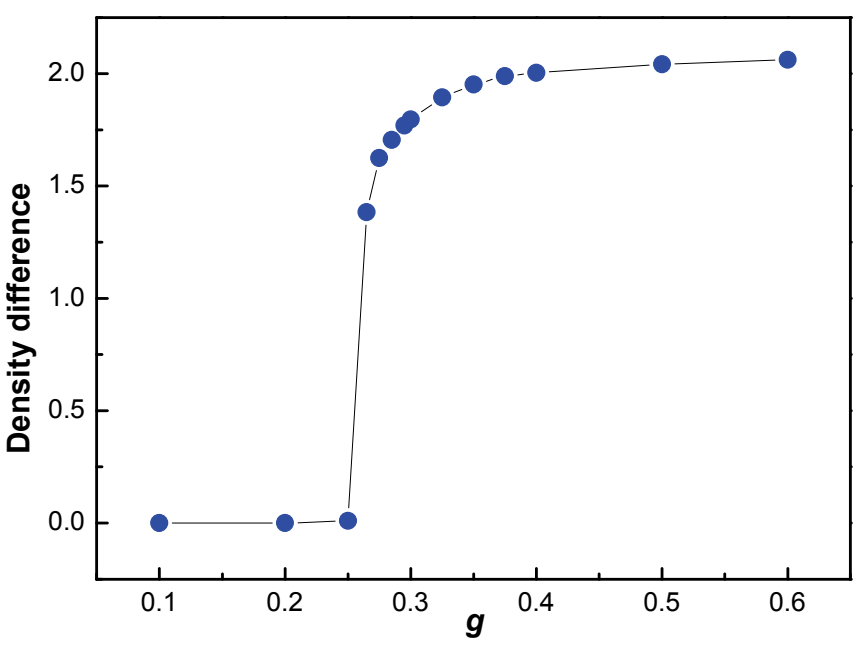

Fig. 4 Density difference of fluid 1(liquid phase) as a function of fluidfluid interaction $g$

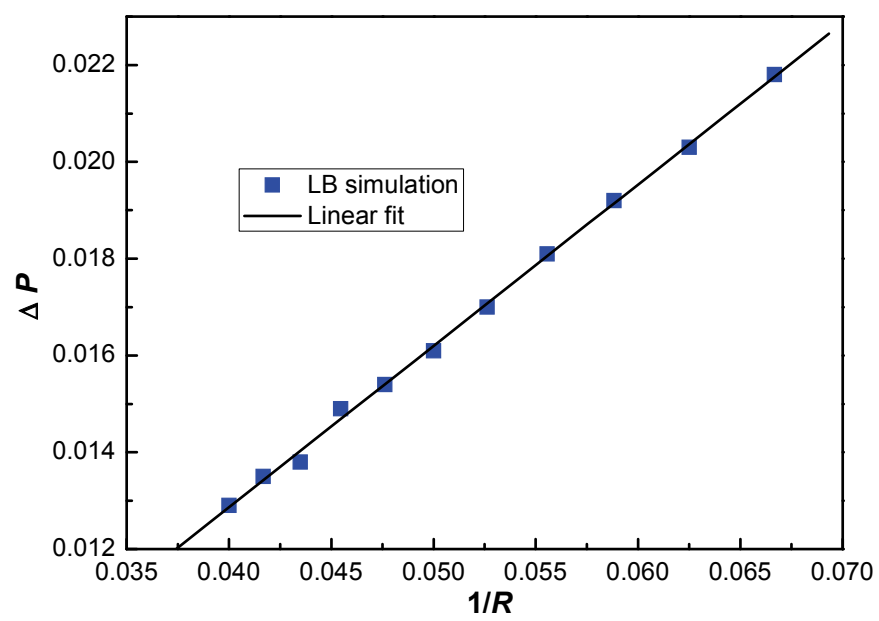

Fig. 5 The pressure difference inside and outside the droplet as a function of the reciprocal of droplet radius $1 / R$ 


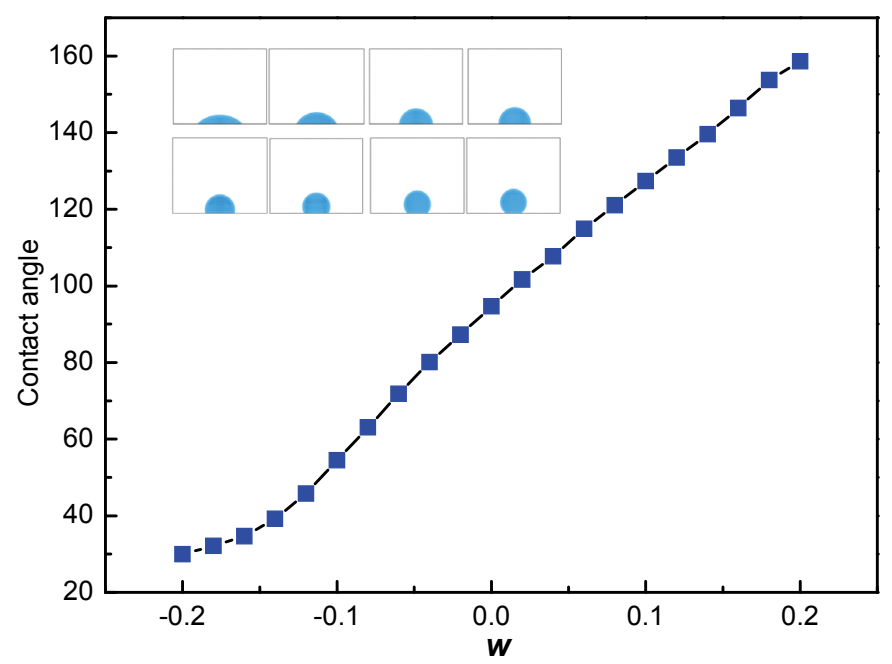

Fig. 6 Contact angle of droplets on the solid surface as a function of fluid-solid interaction $w$

The relationship between measured contact angle and $w$ is presented in Fig.6. Previous literature reported that the contact angle is a linear function of $w$ (Huang, Thorne et al. 2007). Our simulation results show a good agreement with these reports. Hence by adjusting $w$, different contact angle can be obtained. In our simulation of liquid water transport behaviors in GDL nd GC, contact angle is about $120^{\circ}$ for hydrophobic surface and $60^{\circ}$ for hydrophilic surface, respectively. Thus the corresponding $w$ is selected as 0.08 for hydrophobic surface and -0.09 for hydrophilic surface, respectively, according to Fig.6.

\section{RESULTS AND DISCUSSION}

\subsection{Liquid water transport in the GDL and GC}

Figure 7 shows the liquid water transport processes from the inlet to the GDL-GC interface. There is no air flow in the GC as in present paper the emphasis doesn't put on the liquid water behaviors under the air flow. Therefore, the air flow in the GC is set as zero. Initially, the density of air is 2.0 everywhere except the liquid water inlet where the air density is $1.0 \mathrm{E}-5$. The liquid water density is set as 2.0 at the liquid water inlet and 1.0E-5 elsewhere. In all the solid phase of carbon fibers, densities of both air and liquid water are zero. The inlet velocity of liquid water is $8 \times 10^{-5}$ in lattice unit and the corresponding $\mathrm{Ca}$ is $4 \times 10^{-5}$, which is larger than the typical $C a$ in the practical PEMFC operation. However, liquid water behaviors under present $\mathrm{Ca}$ already fall in the regime of capillary fingering as can be observed in Fig. 7. In Fig. 7 the liquid water color is blue, the air color is light yellow and the carbon fibers are dark. Obviously, during transporting inside the GDL, liquid water preferentially chooses the largest pores to invade, which is the typical character of capillary fingering. When the liquid water reaches the GDL-GC interface, a flexuous pathway is formed inside the GDL as can be seen in Fig. 7 (c).

Figure 8 shows the liquid water dynamic behaviors after the liquid water reaches the GDL-GC interface. As there is no carbon fiber in the $\mathrm{GC}$, the liquid water flows and forms a liquid droplet under the dominant liquid-water surface tension in the GC as can be seen in Fig. 8. It also can be observed in the red circles of Fig. 8(a) that there are two possible locations at the GDL-GC interface for the liquid water to form droplets. If looking from the upside of GDL-GC interface, two droplets will be observed as shown in Fig. 9(a) (liquid water under the dashed red line can't be seen as the carbon fiber is opaque). However, once the liquid water preferentially forms a droplet on the left where the pore is larger, the liquid water on the right recedes as shown in Fig. 8(b). Therefore, liquid water on the right dismisses as shown in Fig. 9(b). The droplet on the left continuously grows up and the liquid water on the right gradually recedes as shown in Fig. 8(c) and Fig. 9(c). This phenomenon is similar to the experimental observation which suggesting a dynamic interconnection of water pathways within the GDL (Bazylak, Sinton et al. 2008).

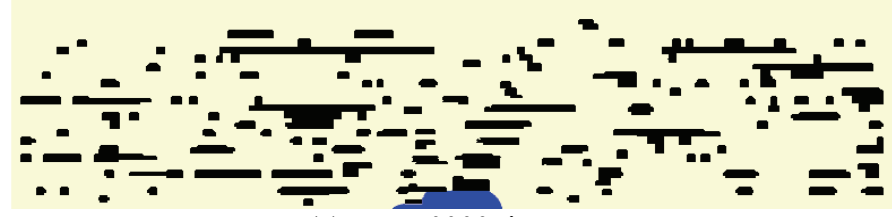

(a) $t=50000$ time step

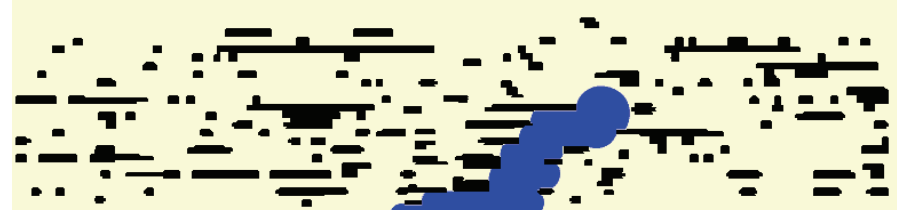

(b) $t=250000$ time step

Fig. 7 Liquid water transport process from inlet to GDL-GC interface at different time steps.

\subsection{Effects of channel land}

In order to investigate the effects of channel land on liquid water transport processes in the GDL and GC, a channel land is added in the GC. The width of the channel land is $300 \mu \mathrm{m}$ and the height is $200 \mu \mathrm{m}$. The channel land is placed at the center of the GC in the $x$ direction. Such placement will cover the GDL-GC interface where the liquid water forms a droplet as discussed in section 4.1. The two side channel surfaces of the channel land and the top surface of the GC is set as hydrophilic while the bottom surface of the channel land and the GDL is hydrophobic. Fig. 10 presents the liquid water dynamic behaviors in the GDL and GC. Compared to liquid water transport processes in GDL and GC without a channel land, liquid water shows the fairly similar behaviors in the GDL as can be seen in Fig. 10(a) to Fig. 10(b). However, the water dynamic behaviors near the GDL-GC interface changes. Because the GDL-GC interface where the droplet is formed in 
the simulation with no channel land is covered by the hydrophobic surface of channel land, the liquid water can't invade there and it has to select the way at the right where it recedes in the simulation with no channel land, as shown in Fig. 10(c). Because the side surface of the channel land is hydrophilic, the initially formed small droplet (Fig. $10(c))$ is attracted by the side surface of channel land and tends to spreads on the side surface as shown in Fig. 10(d).

\subsection{Effects of channel land surface wettabilities}

In order to investigate the effects of channel surface wettabilities on liquid water transport, the side surface of channel land is set as hydrophobic. Other settings are similar to that in section 4.2. Fig. 11 shows the liquid water dynamic behaviors in the GDL and GC. It can be observed that liquid water transport process inside the GDL is also similar to that without channel land. The effects of the channel land are more obvious near the GDL-GC interface and in the GC. It can be observed again that liquid water choose the right way at the GDL-GC interface to enter the GC. Due to the hydrophobic side surface of the channel land, the initially formed small droplet no longer climbs to the channel surface to form water film. Instead, it grows and forms a larger droplet which tends to be apart away from the side surface of channel land.

From the above simulations, it is found that channel land has little influence on the liquid water dynamic behaviors and distribution inside the GDL. However, it can change the water distribution near the GDLGC interface. Moreover, the channel land surface wettabilities greatly affects the liquid water distribution in the GC, which is supported by the simulation results (Zhu, Sui et al. 2008).

\subsection{Effects of channel land location}

In order to investigate the effects of channel land location on liquid water dynamic behaviors and distributions in the GDL and GC, the channel land is rearranged in the GC as shown in Fig. 12 and Fig. 13. The channel land is placed from $x=50 \mu \mathrm{m}$ to $x=350 \mu \mathrm{m}$ along the $x$ direction. Such arrangement will not cover the GDL-GC interface where the liquid water forms a droplet in the simulation without channel land. In Fig. 12, the surface wettabilities is similar to the simulation in section 4.2. In Fig. 13, the surface wettabilities is similar to the simulation in section 4.3.

It can be observed from the set of Fig. 12 and Fig. 13 that if the channel land is away from the GDL-GC interface where the liquid droplet forms in the simulation without a channel land, the channel land has little influences on the liquid water transport behaviors in the GC as will as in the GC. The liquid water dynamic behaviors and final distributions are almost the same as that in the GDL and GC without channel land. Of course, liquid water from the CL can arrive at the GDL-GC interface under the channel land as well as the GDL-GC interface under the open GC. Therefore, in the GC with hydrophilic surface channel land, liquid water may presents as films or droplet, depending on the emergence location of the liquid water at the GDLGC interface. For the hydrophobic case, liquid water will always forms droplets, wheresoever the liquid water from hydrophobic GDL emergences. Compared Fig.7 to Fig. 13, It should be noted that the time needed for liquid water from inlet into the $\mathrm{GC}$ is different. The time needed for liquid water transport from the inlet to the GC corresponding to the cases with hydrophilic channel land is shorter than that without channel land in the GC and that with a hydrophobic channel land in the GC. It seems that a hydrophilic channel land tends to accelerate the liquid water transport process inside the GDL while maintains the liquid water transport mechanism as capillary fingering. At present it is not sure whether this is indeed the case in an operating PEMFC or it is the artificial results of the numerical model, as current experimental techniques which suffer the drawbacks of temporal limitations can't give the whole micro-scale dynamic processes from GDL-CL interface to the GDL-GC interface.

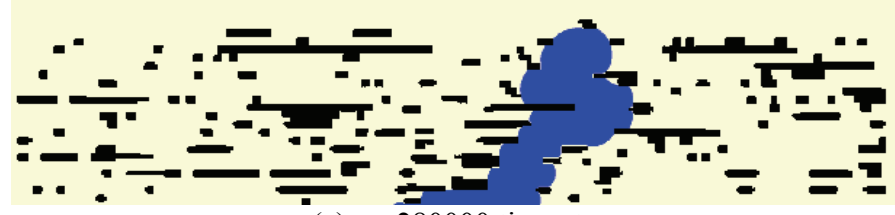

(c) $t=280000$ time step

Fig. 7 (continued).

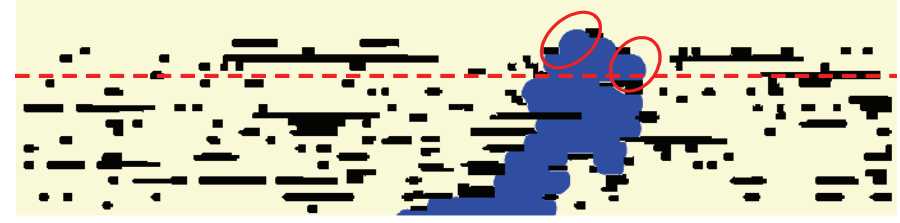

(a) $t=310000$ time step

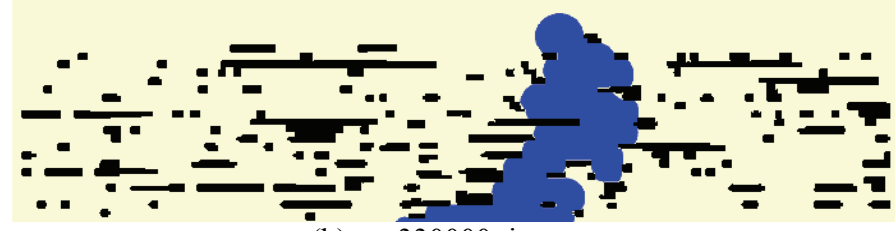

(b) $t=330000$ time step 


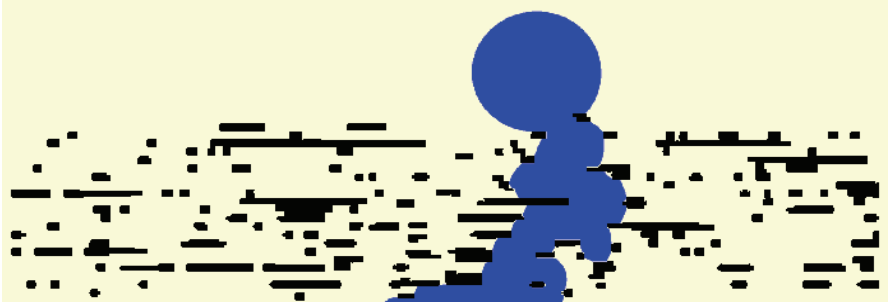

(c) $t=370000$ time step

Fig. 8 Liquid water transport process near the GDL-GC interface and in the GC at different time steps.

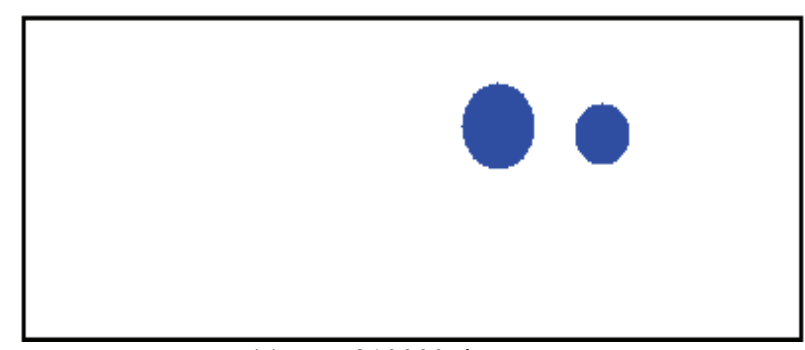

(a) $t=310000$ time step

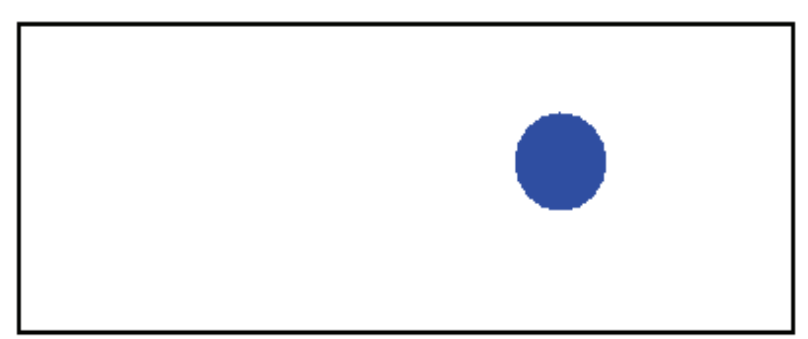

(b) $t=330000$ time step

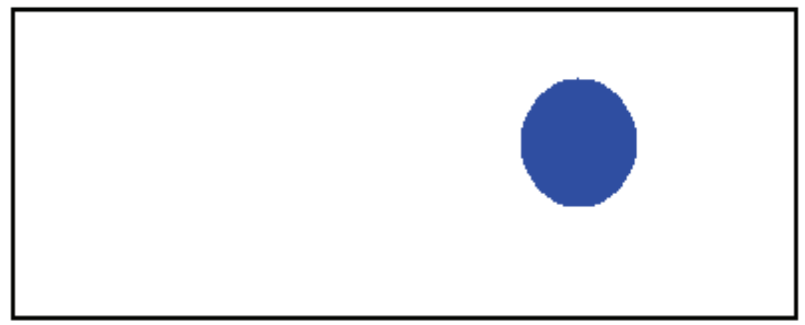

(c) $t=370000$ time step

Fig. 9 Liquid water images observed from the upside of the GDL-GC interface at different time

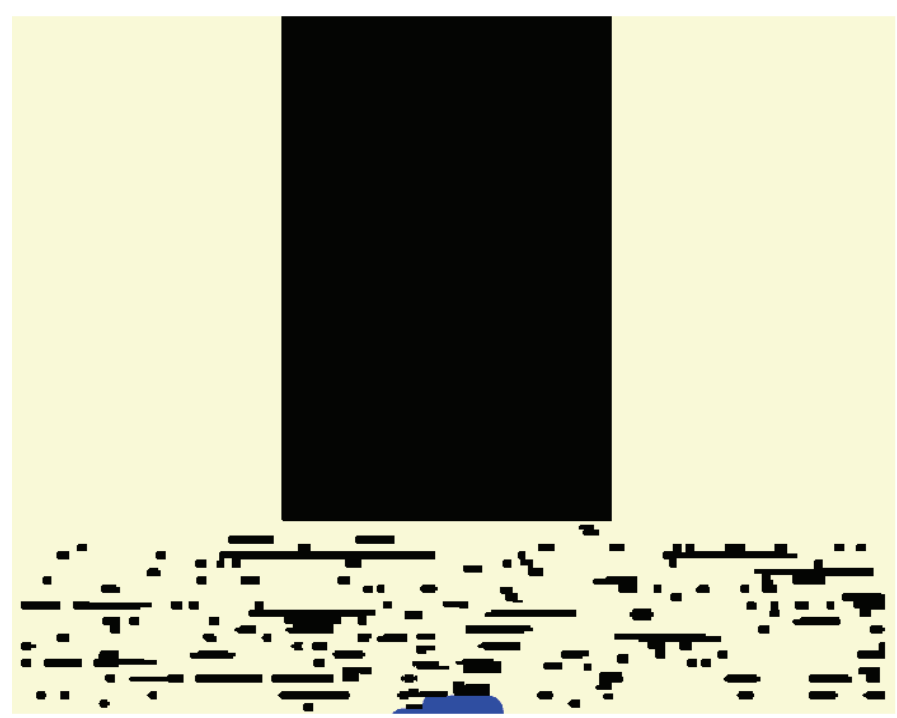

(a) $t=50000$ time step

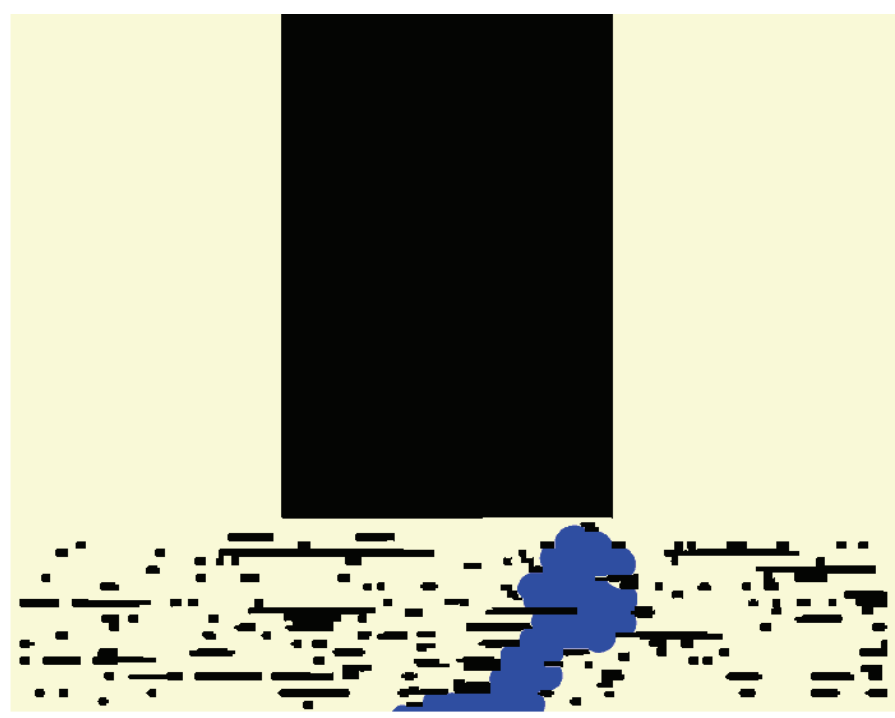

(b) $t=160000$ time step

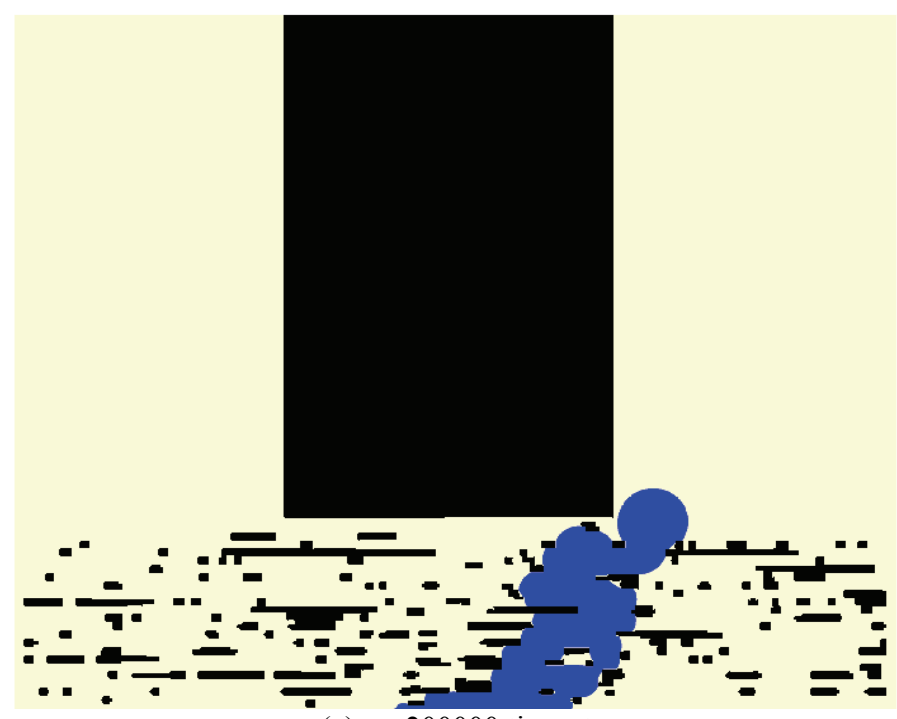

(c) $t=200000$ time step 


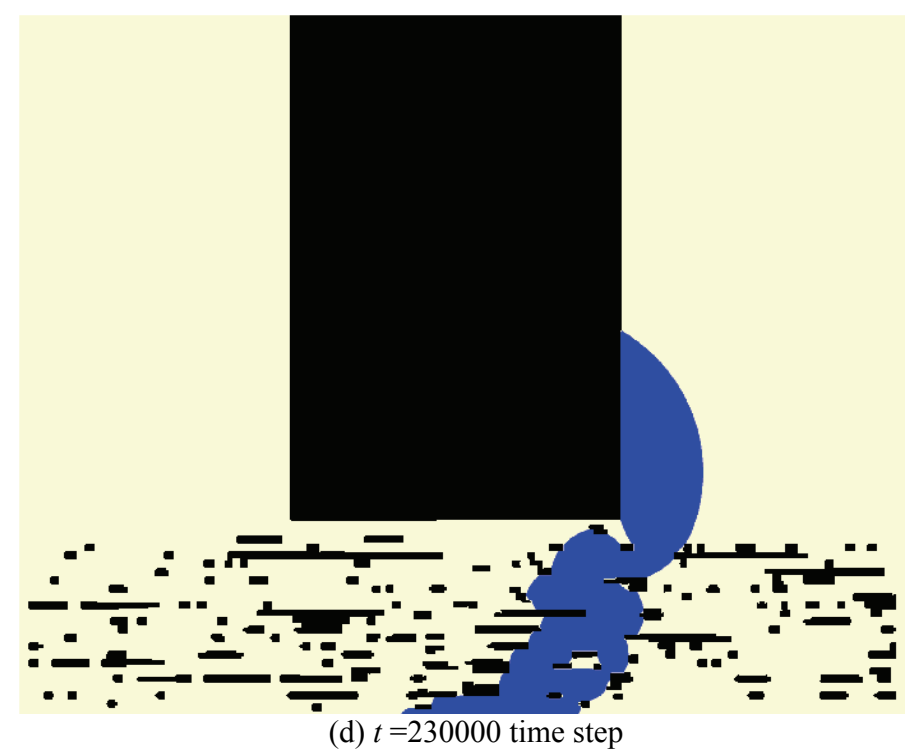

Fig. 10 Liquid water transport process in the GDL and GC with a hydrophilic channel land

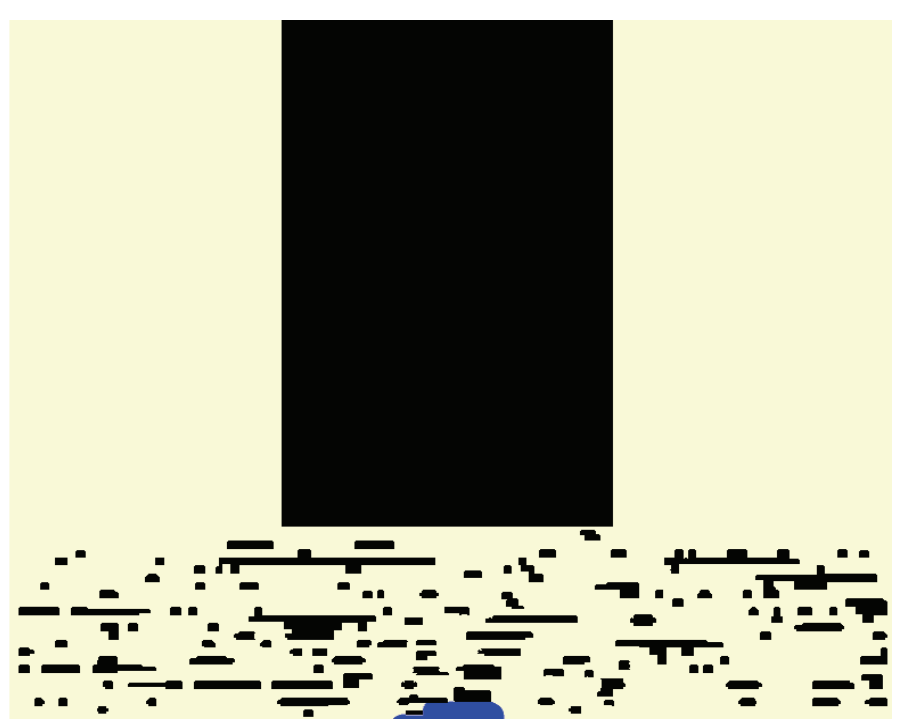

(a) $t=100000$ time step

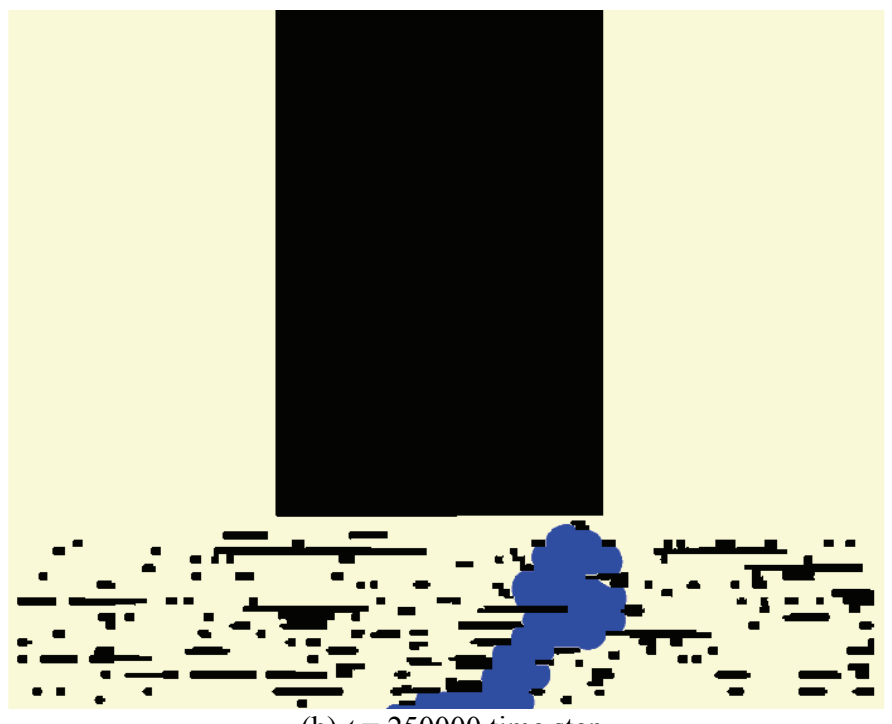

(b) $t=250000$ time step

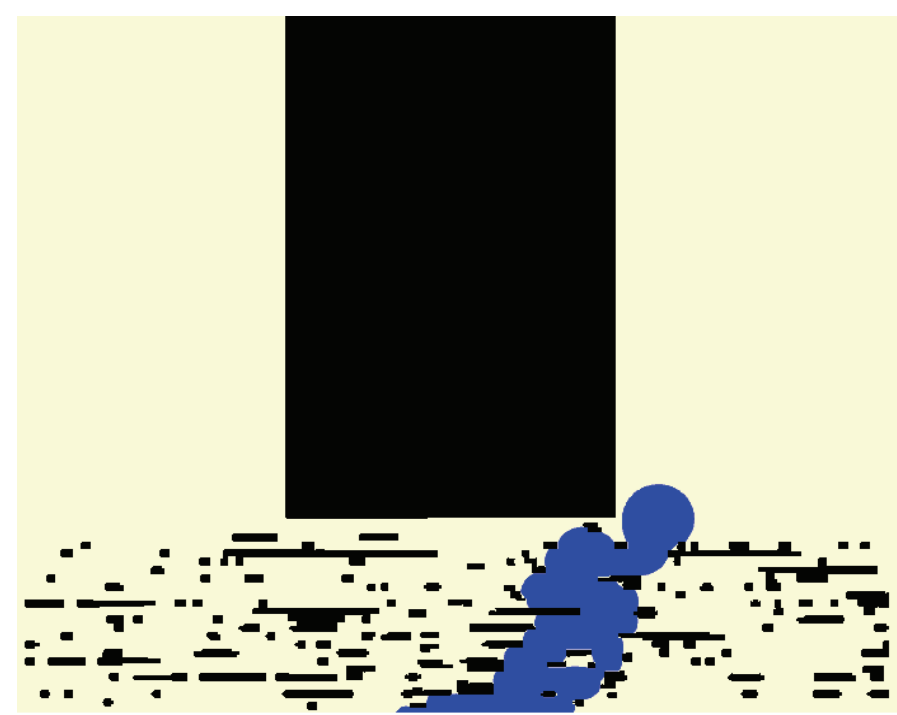

(c) $t=330000$ time step

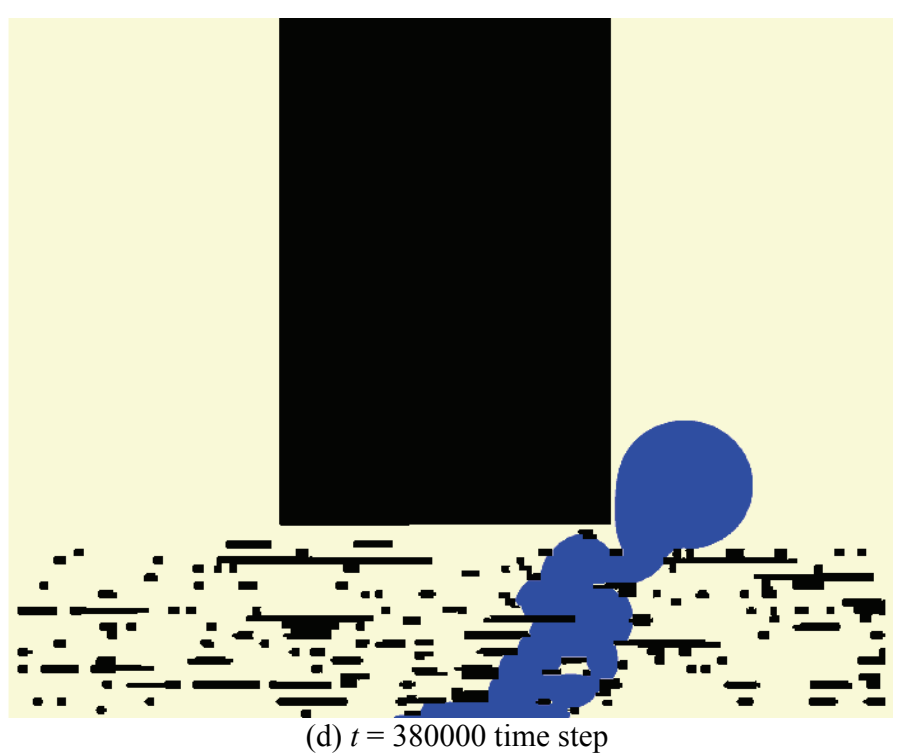

Fig. 11 Liquid water transport process in the GDL and GC with a hydrophobic channel land at different time

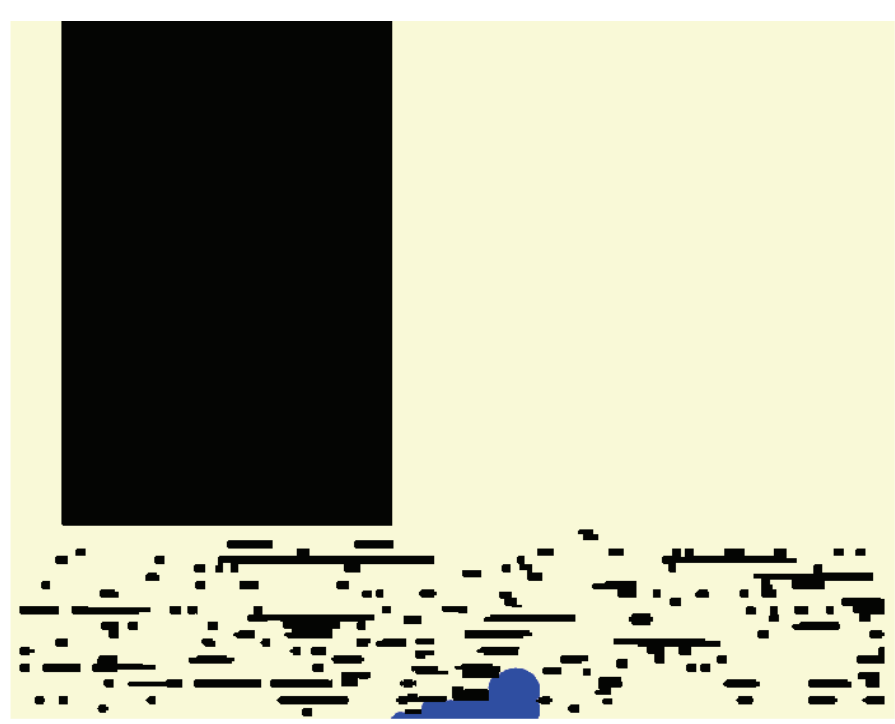

(a) $t=100000$ time step 

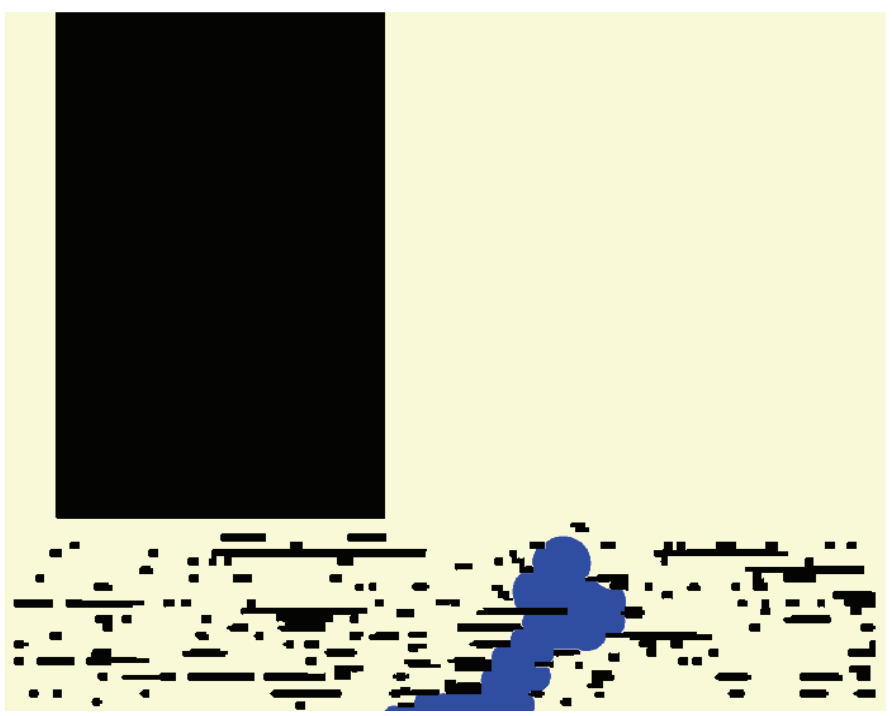

(b) $t=150000$ time step

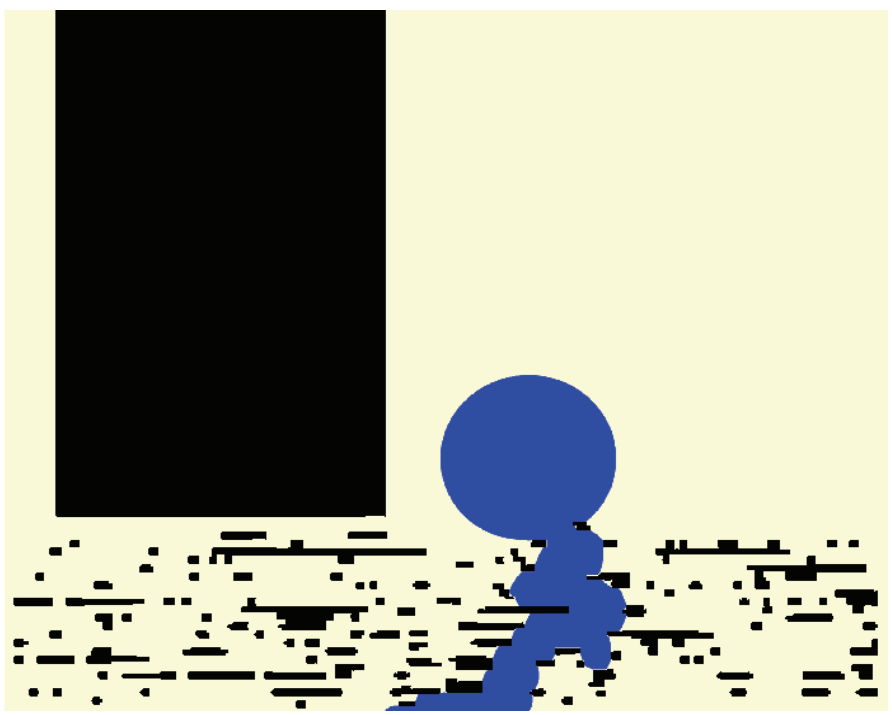

(c) $t=250000$ time step

Fig. 12 Liquid water transport process in the GDL and GC with a hydrophilic channel land. The channel land is rearranged

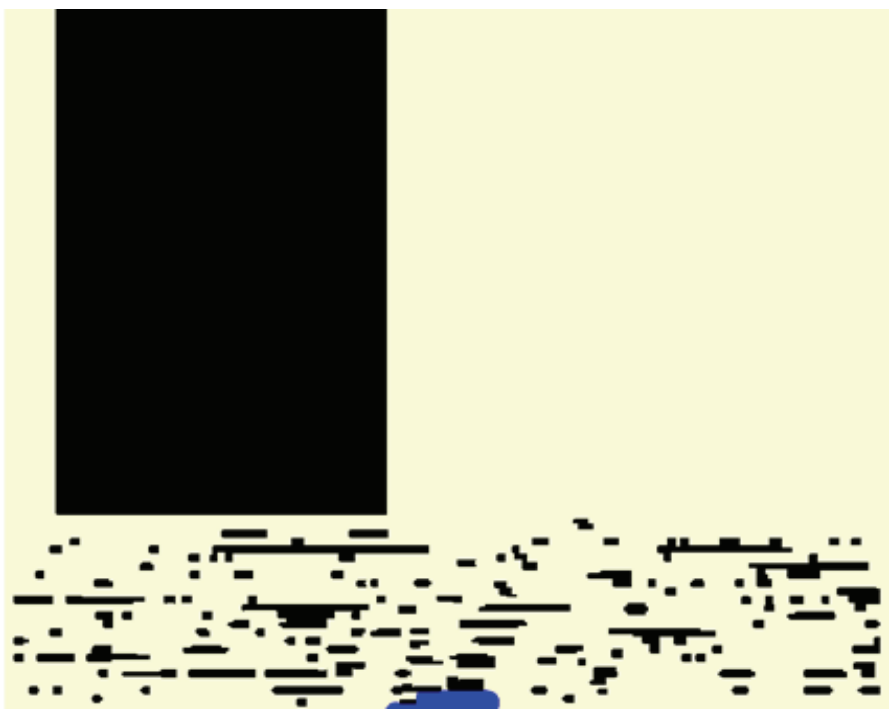

(a) $t=150000$ time step
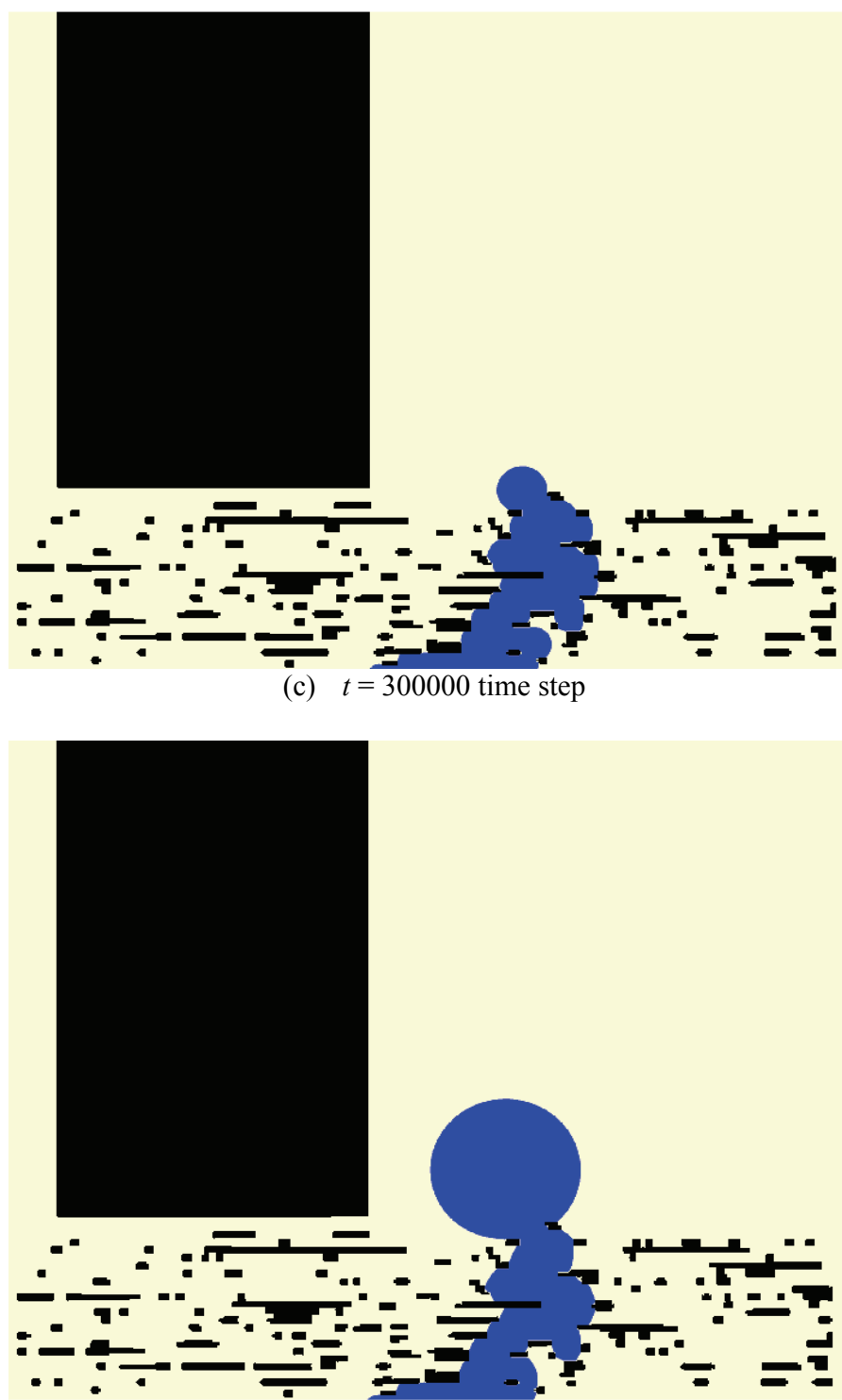

(c) $t=380000$ time step

Fig. 13 Liquid water transport process in the GDL and GC with a hydrophobic channel land. The channel land is rearranged

\section{Conclusion}

In this paper, the multi-phase lattice Boltzmann method (SC model) is used to investigate the liquid water dynamic behaviors and distributions in the GDL and GC. Firstly the SC model is used to simulate liquid water transport process in the GDL and GC without channel land. It is found that liquid water transport process is capillary fingering. At the GDL-GC interface, due to the dominant water-air surface tension force, a droplet is formed. The water dynamic behaviors near the GDL-GC indicate the dynamic interconnection of liquid water pathways in the GDL. Then a channel land is added in the GC to investigate the effects of channel land surface wettabilities and channel land location on liquid water dynamic behaviors. When the channel land covers the GDL-GC interface where liquid water forms a droplet in the GC without channel land, the channel land changes the water distribution near the GDL-GC interface and in the GC. The liquid water tends to spread and present as film on the hydrophilic side surface of channel land. For the case with hydrophobic channel land side surface, the liquid water tends to form a droplet which is apart from the side surface. Then the channel land is rearranged and doesn't 
cover the interface where the liquid water forms a droplet in the GC without channel land, it is found that the channel land has little influence on the liquid water distribution in the GDL and GC. It is also found that a hydrophilic channel land helps to reduce the time needed for liquid water transporting from GDL-CL interface to GDL-CL interface, which is uncertain whether indeed the case or is the artificial results of the numerical model.

\section{ACKNOWLEDGEMENTS}

The authors thank the National Nature Science Foundation of China (No.50636050) for supporting this work.

\section{NOMENCLATURE}

$\begin{array}{ll}c & \Delta x / \Delta t \\ C a & \text { Capillary number } \\ c_{s} & \text { sound velocity in lattice unit } \\ \boldsymbol{e}_{i} & \text { lattice discrete velocity } \\ f & \text { particle distribution function } \\ G & \text { Green function } \\ g & \text { fluid-fluid interaction in SC model } \\ P_{i} & \text { pressure inside the droplet } \\ P_{o} & \text { pressure outside the droplet } \\ R & \text { radius of droplet in lattice unit } \\ t & \text { time in lattice unit } \\ \boldsymbol{u} & \text { velocity in lattice unit } \\ W & \text { Green function } \\ w & \text { fluid-solid interaction in SC model } \\ \text { Greek Symbols } \\ \rho & \text { density in lattice unit } \\ \sigma & \text { surface tension in lattice unit } \\ \tau & \text { relaxation time in LBM } \\ \psi & \text { effective density } \\ \text { Superscripts } & \\ i & \text { ith discrete direction for LBM model } \\ \text { Subscripts } & \\ \text { eq } & \text { equilibrium condition } \\ k & k \text { th component }\end{array}$

\section{REFERENCES}

Bazylak, A., 2009, "Liquid water visualization in PEM fuel cells: A review," International Journal of Hydrogen Energy, 34(9), 3845-3857.

doi:10.1016/j.ijhydene.2009.02.084

Bazylak, A., Sinton, D. and Djilali, N., 2008, "Dynamic water transport and droplet emergence in PEMFC gas diffusion layers," Journal of Power Sources, 176(1), 240-246.

doi:10.1016/j.jpowsour.2007.10.066

Chen, S.Y. and Doolen, G.D., 1998, "Lattice Boltzmann methode for fluid flows," Annual Review of Fluid Mechanics, 30, 329-364.

doi:10.1146/annurev.fluid.30.1.329

FAL., D., 1992, "Porous media: fluid transport and pore structure," San Diego: Academic Press;.

Gostick, J.T., Fowler, M.W., Ioannidis, M.A., Pritzker, M.D., Volfkovich, Y.M. and Sakars, A., 2006, "Capillary pressure and hydrophilic porosity in gas diffusion layers for polymer electrolyte fuel cells," Journal of Power Sources, 156(2), 375387.

doi:10.1016/i.jpowsour.2005.05.086

Hao, L. and Cheng, P., 2009, "Lattice Boltzmann simulations of anisotropic permeabilities in carbon paper gas diffusion layers," Journal of Power Sources, 186(1), 104-114.

doi:10.1016/j.jpowsour.2008.09.086

Huang, H., Thorne, D.T., Schaap, M.G. and Sukop, M.C., 2007, "Proposed approximation for contact angles in Shan-and-Chentype multicomponent multiphase lattice Boltzmann models," Physical Review E, 76(6), 066701.

dol:10.1103/PhysRevE.76.066701

Koido, T., Furusawa, T. and Moriyama, K., 2008, "An approach to modeling two-phase transport in the gas diffusion layer of a proton exchange membrane fuel cell," Journal of Power Sources, 175(1), 127-136.

doi:10.1016/j.jpowsour.2007.09.029

Lee, K.J., Nam, J.H. and Kim, C.J., 2009, "Pore-network analysis of two-phase water transport in gas diffusion layers of polymer electrolyte membrane fuel cells," Electrochimica Acta, 54(4), 1166-1176.

doi:10.1016/j.electacta.2008.08.068

Li, H., Tang, Y., Wang, Z., Shi, Z., Wu, S., Song, D., Zhang, J., Fatih, K., Zhang, J., Wang, H., Liu, Z., Abouatallah, R. and Mazza, A., 2008, "A review of water flooding issues in the proton exchange membrane fuel cell," Journal of Power Sources, 178(1), 103-117.

doi:10.1016/j.jpowsour.2007.12.068

Martys, N.S. and Chen, H., 1996, "Simulation of multicomponent fluids in complex three-dimensional geometries by the lattice Boltzmann method," Physical Review E, 53(1), 743-750.

doi:10.1103/PhysRevE.53.743

Mukherjee, P.P., Wang, C.Y. and Kang, Q., 2009, "Mesoscopic modeling of two-phase behavior and flooding phenomena in polymer electrolyte fuel cells," Electrochimica Acta, 54(27), 6861-6875.

doi:10.1016/j.electacta.2009.06.066

Nam, J.H. and Kaviany, M., 2003, "Effective diffusivity and water-saturation distribution in single- and two-layer PEMFC diffusion medium," International Journal of Heat and Mass Transfer, 46(24), 4595-4611. doi:10.1016/S0017-9310(03)00305-3

Natarajan, D. and Nguyen, T.V., 2001, "A Two-Dimensional, Two-Phase, Multicomponent, Transient Model for the Cathode of a Proton Exchange Membrane Fuel Cell Using Conventional Gas Distributors," J. Electrochem. Soc., 148, A1324 - A1335. Permalink: http://dx.doi.org/10.1149/1.1415032

Nguyen, P.T., Berning, T. and Djilali, N., 2004, "Computational 
model of a PEM fuel cell with serpentine gas flow channels," Journal of Power Sources, 130(1-2), 149-157.

doi:10.1016/j.jpowsour.2003.12.027

Nguyen, T.V., 1996, "A gas distributor design for protonexchange membrane fuel cells," J. Electrochem. Soc., 143, L103-L105.

Permalink: http://dx.doi.org/10.1149/1.1836666

Park, J. and Li, X., 2008, "Multi-phase micro-scale flow simulation in the electrodes of a PEM fuel cell by lattice Boltzmann method," Journal of Power Sources, 178(1), 248257.

doi:10.1016/j.jpowsour.2007.12.008

Park, J., Matsubara, M. and Li, X., 2007, "Application of lattice Boltzmann method to a micro-scale flow simulation in the porous electrode of a PEM fuel cell," Journal of Power Sources, 173(1), 404-414.

doi:10.1016/j.jpowsour.2007.04.021

Puneet K. Sinha, P.Mukherjee, P. and Wang, C.Y., 2007, "Impact of GDL structure and wettability on water management in polymer electrolyte fuel cells," J. Mater. Chem., , 17(30), $3089-3103$. doi: $10.1039 / \mathrm{b} 703485 \mathrm{~g}$

Shan, X. and Chen, H., 1993, "Lattice Boltzmann model for simulating flows with multiple phases and components," Phys. Rev. E, 47, 1815-1819.

doi:10.1103/PhysRevE.47.1815

Shan, X. and Chen, H., 1996, "Diffusion in a multicomponent lattice Boltzmann equation model," Phys. Rev. E, 47, 36143620 .

doi:10.1103/PhysRevE.54.3614

Shan, X. and Doolen, G., 1995, "Multicomponent lattice Boltzmann model with inter particle interaction," Journal of
Statistical Physics, 81, 379-393.

doi:10.1007/BF02179985

Siegel, N.P., Ellis, M.W., Nelson, D.J. and von Spakovsky, M.R., 2004, "A two-dimensional computational model of a PEMFC with liquid water transport," Journal of Power Sources, 128(2), 173-184.

doi:10.1016/j.jpowsour.2003.09.072

Wang, Z.H., Wang, C.Y. and Chen, K.S., 2001, "Two-phase flow and transport in the air cathode of proton exchange membrane fuel cells," Journal of Power Sources, 94(1), 40-50. doi:10.1016/S0378-7753(00)00662-5

Wilson, M.S., Valerio, J.A. and Gottesfeld, S., 1995, "Low platinum loading electrodes for polymer electrolyte fuel cells fabricated using thermoplastic ionomers," Electrochimica Acta, 40(3), 355-363.

doi:10.1016/0013-4686(94)00272-3

You, L. and Liu, H., 2002, "A two-phase flow and transport model for the cathode of PEM fuel cells," International Journal of Heat and Mass Transfer, 45(11), 2277-2287.

doi:10.1016/S0017-9310(01)00322-2

Zhu, X., Sui, P.C. and Djilali, N., 2008, "Three-dimensional numerical simulations of water droplet dynamics in a PEMFC gas channel," Journal of Power Sources, 181(1), 101-115.

doi:10.1016/j.jpowsour.2008.03.005

Ziegler, D.P., 1993, "Boundary conditions for lattice Boltzmann simulations," 71, 1171-1177.

doi:10.1007/BF01049965

Zou, Q.S. and He, X.Y., 1997, "On pressure and velocity boundary condition for lattice Boltzmann BGK model," Physicas of fluids, 9, 1591-1598.

doi:10.1063/1.869307 